\title{
Exploration of Current Trend on Median Filtering Methods Utilized in Digital Grayscale Image Processing
}

\author{
Sin Hoong Teoh, Boon Tatt Koik, and Haidi Ibrahim
}

\begin{abstract}
Median filtering is a well known method to deal with impulse noise in digital images. However, due to some limitations associated with the standard median filtering approach, several new improved versions of the median filtering method have been proposed by researchers. The number of methods is expanding from year to year. Therefore, the purpose of this paper is to see the current trend of the median filtering techniques. The trend is observed from the available online literatures; which use or propose new median filtering methods. Because there are thousands of related reliable literatures available online alone, our research concentrates on online literatures only. These literatures were classified according to their search keywords. The scope of our research is limited to IEEExplore ${ }^{\circledR}$, ScienceDirect, and Google Scholar databases only. The results confirm that the research regarding to median filtering is still growing at this time. Furthermore, we observed that the weighted median filter is the most popular median filtering research, and it is followed by researches on the adaptive median filter. Based on ScienceDirect database, it is shown that all of the median filtering methods showing an increasing number of publications over year.
\end{abstract}

Index Terms-Impulse noise, literature survey, median filtering, salt-and-pepper noise.

\section{INTRODUCTION}

As the demand of the products from the consumers are showing an increasing trend, most of the industries are now employing automatic electronic systems in their production lines. Computerized systems, utilizing optical sensors such as CCD and CMOS, becoming popular in recent years [1]. In order to reduce the burden of human operators in inspection lines, machine vision systems must simulate the human abilities to detect defect products. Therefore, to imitate and put into practice the function of human vision system, the computerized system must use engineering methods, especially from the field of computer, physics and electronic engineering. However, it is worth noting that up to these date, these vision systems are mostly used to assist the work by the human operators, and not completely replacing the human in the production line [2].

Signal acquired by the machine vision system is mostly in the form of digital images, which are two or more dimensional signals. One type of digital images used by the system is the digital grayscale image [3]. Unfortunately, similar to other type of digital images, digital grayscale

Manuscript received December 5, 2012; revised February 6, 2013.

The authors are with the School of Electrical \& Electronic Engineering, Engineering Campus, Universiti Sains Malaysia, 14300 Nibong Tebal, Penang, Malaysia (e-mail: tshoong81@ yahoo.com, bt5041@gmail.com, haidi_ibrahim@ieee.org). images can be corrupted by unnecessary noise signals, including the impulse noise [4]. There are some reasons how the impulse noise can appears in digital grayscale images. Impulse noise can be contributed by malfunctioned image sensors, damaged memory cell in hard disk, and signal corruption during transmission [5]. Impulse noise is a random additive noise, and it has significantly high contrasts towards its surrounding, uncorrupted region. Therefore, even at a very low corruption level of impulse noise, the digital grayscale image might loss its information significantly [6]. As a consequence, the noise mostly will make the automatic vision based systems to give inaccurate outcomes [7].

One of the methods that are used widely to deal with impulse noise in digital grayscale images is the median filtering method [4]. Standard median filtering method works by using a sliding window. It filters the corrupted input image by replacing the corresponding pixel that located on the centre of the window, with the median value determined from the intensity samples, defined by the sliding window at that position [8]. Unfortunately, the standard median filtering method uses all the intensity samples within the sliding window, without considering whether they are noisy pixels, and therefore produce inaccurate results when the input image was damaged by a high degree of impulse noise [9]. On the other hand, as the method changes all the pixel values although the pixels are uncorrupted, the method tends to make unnecessary changes. Therefore, the method will further degrade the image, especially when the input image was corrupted by low level of impulse noise [5]. Furthermore, sharp lines also will be altered by the standard median filter [10].

The limitations of the standard median filter have attracted attention from researchers. In order to overcome these limitations of the standard median filter, many researchers have proposed an extension, or improvement to the method. Hundreds of reliable literatures based on median filtering methods can be found online these days. We believe that there are another hundreds of related literatures that are not available online. According to our past literature survey on the available median filtering method [11], in addition to the standard median filtering method, the improved median filtering methods can be classified into seven categories. They are:

1) Weighted median filter

- First introduced by Justusson in 1981, and further elaborated by Brownrigg [12].

- It has weights to associate with each of its filter element.

- The weight corresponds to the number of sample 
duplications in the calculation of median value.

2) Iterative median filter

- Iterative method requires the same procedure to be repeated several times.

- Examples are methods in [13]-[15].

3) Recursive median filter

- The output at certain position are determined not only using the input intensities, but also from the pre-calculated output values at previous locations.

- Analogous to infinite impulse response (IIR) filter

- Examples of related methods are [16]-[18].

- Directional median filter

- Works by separating its 2-D sliding window into several 1-D filter components.

- Examples of works are [19]-[21].

4) Switching median filter

- Also known as decision based median filter.

- Normally has two stages; noise detection stage, and noise cancellation stage

- Example of works are [22]-[24].

5) Adaptive median filter

- The filter size is not constant.

- The size is depending to local noise content.

- Examples of works are [25]-[27].

6) Median filter with fuzzy logics

- Uses fuzzy logic to process the image.

- Example of works are [28]-[30].

Each of these abovementioned approaches has its own advantages and disadvantages. As consequences, more and more new methods have been introduced from time to time.

It is worth noting that median filtering method is one of the fundamental methods in digital image processing research. Yet, most of the people, especially those who are outside the field of digital image de-noising, will be surprised when we mentioned that the researches on median filtering technique are still popular these days. Therefore, the aim of this research paper is to show quantitatively that the research on median filtering methods is still expanding. In addition to this, we are also interested to see the current research trend in this area. We want to identify which median filtering approach is now popular, and the trend of the median filtering research towards time.

To ease the presentation of this paper, this paper is organized into four main sections. The first section, which is this section, gives the background and the purposes of this research. Then, Section II explains how this research will be carried out. Section III presents the outcome of this research, while the last section, which is Section IV, concludes our findings.

\section{METHODOLOGY}

Currently, the number of researches regarding to median filtering for impulse noise reduction from grayscale digital images is very large. Search on internet alone, regarding median filtering method, gave us hundreds of related reliable literatures. As a consequence, it is almost impossible for us to inspect every single literature in details for the methodology they used or proposed.

To overcome this limitation, we will classify these literatures based on their keywords. We assume that the keywords are used by the literatures reflects exactly the median filtering approach used by that literature. The keywords used are listed in Table I.

TABLE I: THE KEYWORDS USED TO SEARCH RELATED LITERATURES

\begin{tabular}{ll}
\hline \hline Method & Keyword \\
\hline Weighted Median Filter & "Weighted Median Filter Image" \\
Iterative Median Filter & "Iterative Median Filter Image" \\
Recursive Median Filter & "Recursive Median Filter Image" \\
Directional Median Filter & "Directional Median Filter Image" \\
Switching Median Filter & "Switching Median Filter Image" \\
Adaptive Median Filter & "Adaptive Median Filter Image" \\
Median Filter with Fuzzy Logics & "Fuzzy Median Filter Image" \\
Median Filter (in general) & "Median Filter Image" \\
\hline \hline
\end{tabular}

We only restricted our research to online literatures. Because there are many online databases are now available, we are then further limiting our searching to these following three well-known databases:

1) IEEExplore ${ }^{\circledR}$ (http://ieeexplore.ieee.org)

- Is a well known online database regarding to the researches on electrical, electronic and computer engineering.

2) ScienceDirect (http://www.sciencedirect.com)

- Is one of the world's famous database for scientific, technical, and medical full text research papers.

3) Google Scholar (http://scholar.google.com.my)

- Provides a search of literatures across many disciplines and sources, including journals, proceedings, abstracts, books, theses, and patents.

In order to see the research trend of each particular median filtering method, the publication year's field, located on the left side of the corresponding webpage will be manipulated. Example of this field, taken from IEEExplore ${ }^{\circledR}$ database, is shown in Fig. 1. Using this useful feature, the number of publication for each year can be determined. Thus, by recording the number of publications versus years, we can see whether the research is expanding, or shrinking. The results then can represent the popularity of each method. The research has been carried out on early December 2012.

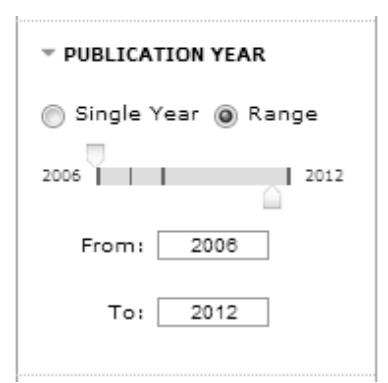

Fig. 1. "Publication Year" feature provided by IEEExplore ${ }^{\circledR}$

\section{RESUlts}

In order to see whether the research regarding median filtering in digital image processing is still popular or not, we utilized the keyword "median filter image" for our search. 
The results from three databases are shown in Fig. 2 to Fig. 5. We cannot combine these results together and present it as one figure because the number of publications obtained from each database is significantly very different from each other.

As shown by these figures, the number of publications on median filtering research is the smallest from IEEExplore $\AA$ database, followed by ScienceDirect, and Google Scholar. This is because the content of IEEExplore ${ }^{\circledR}$ database is only restricted towards electrical, electronic, and computer engineering researches.

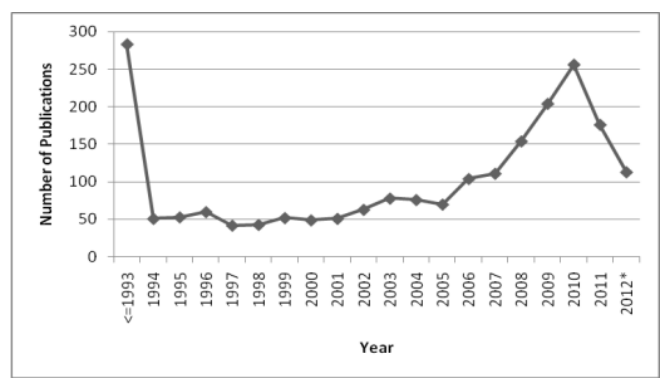

Fig. 2. The trend on the researches regarding to median filtering method, observed from IEEExplore ${ }^{\circledR}$ database.

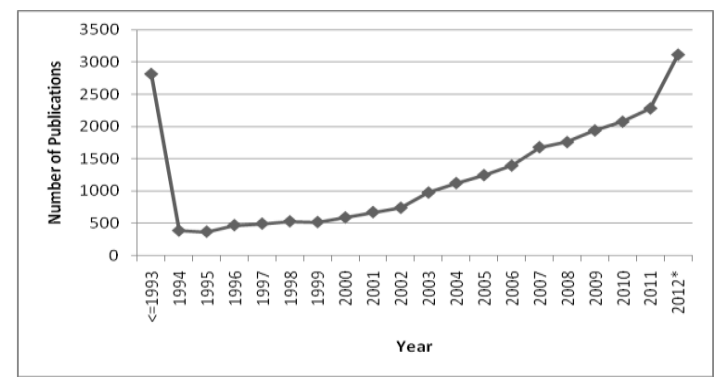

Fig. 3. The trend on the researches regarding to median filtering method, observed from ScienceDirect database.

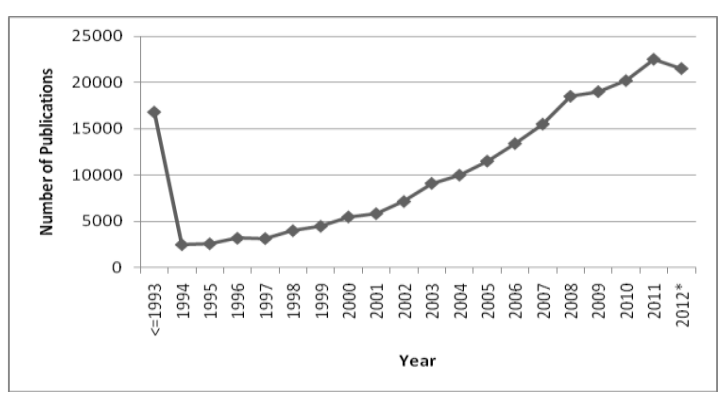

Fig. 4. The trend on the researches regarding to median filtering method, observed from Google Scholar database.

From Fig. 2, it is shows that in IEEExplore database, the research regarding to median filtering is expanding, until 2010, where the rate of the research is slightly reducing after that. However, as shown by Fig. 3 and Fig. 4, the corresponding researches in ScienceDirect and Google Scholar are still expanding. Furthermore, from these figures, we can see that there are more than thousands of related papers introduced in each year. Therefore, this showing that the research related to median filtering method is still expanding these days.

In order to investigate the recent popularity each median filtering method considered in this research, we inputted the keywords shown in Table I to those three databases. By assuming that there is no re-occurrence of literatures between these databases, we plot the accumulated number of publications versus median filtering methods. Because we want to see the current popularity, our search is restricted to year 2000 and beyond. The result is shown by Fig. 5. We assume that the higher number of publications indicates the popularity. Therefore, from this bar graph, we can say that the weighted median filter has the highest popularity, followed by the adaptive median filter, iterative median filter, directional median filter, switching median filter, fuzzy median filter, and lastly, recursive median filter.

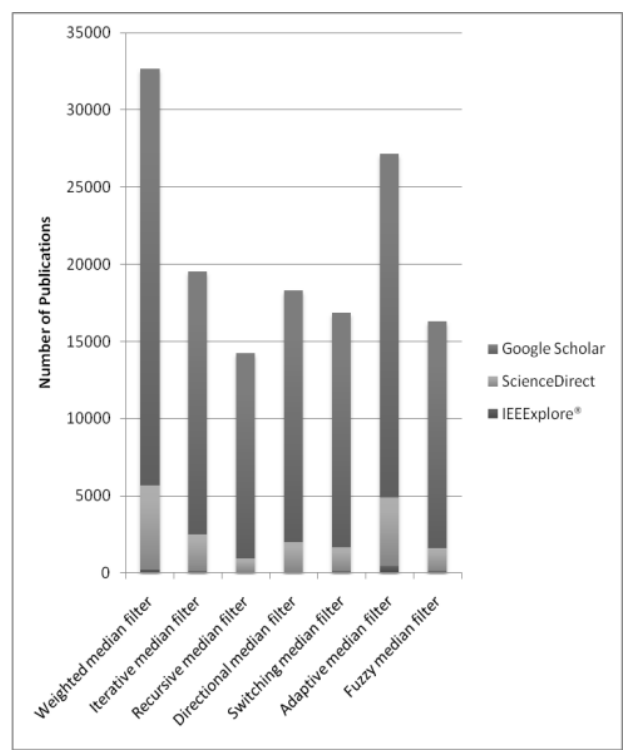

Fig. 5. The bar graph showing the accumulated number of publications versus median filtering methods.

Another question that we want to answer is related to the research trend on each of the median filtering techniques. Therefore, we inspect the trend from ScienceDirect database. The plot of graphs for each median filtering technique, as a function of number of publications versus years was obtained from year 2000 to 2012. This is obtained by utilizing the publication year feature in ScienceDirect. The result is shown in Fig. 6. As shown by this figure, basically all of the methods are showing an increasing in number of publications over years.

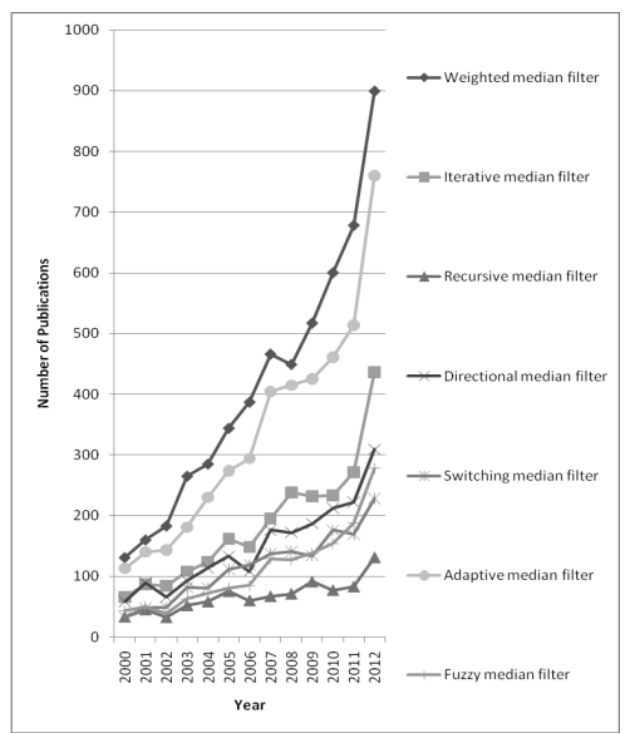

Fig. 6. The trend on the research regarding to each median filtering methods, observed from ScienceDirect database. 


\section{CONCLUSION}

The results from this survey prove that the researches regarding to median filtering in digital image processing field are still expanding. Majority of the online databases shows an exponentially growth of literatures related to this type of research. Based on the trend observed, the number of materials is expected to continue expanding in the future. The research also successfully shows that the trend for each of the median filtering framework is also expanding as well. It is shown that the weighted median filter is the most popular median filtering framework in current literatures, followed by adaptive median filter, iterative median filter, directional median filter, fuzzy median filter, switching median filter, and lastly recursive median filter.

\section{ACKNOWLEDGMENT}

This work was supported in part by the Universiti Sains Malaysia's Short Term Research Grant with account number 304/PELECT/60311013 and Universiti Sains Malaysia's Research University Individual (RUI) Grant with account number 1001/PELECT/814169.

\section{REFERENCES}

[1] C. Y. Wang and D. Lee, "Integration of manufacturing systems: A vision and image processing system perspective," in Proc. of System Integration, 1990, pp. 310-316.

[2] L. Han, F. Xue, Z. Li, and D. Chen, "A generic framework for human-machine hybrid recognition based on wearable visual computing," Applied Mechanics and Materials, vol. 39, pp. 317-321, 2011.

[3] R. C. Gonzalez and R. E. Woods, Digital Image Processing, Upper Saddle River, New Jersey, USA, 2008, ch. 2.

[4] R. C. Gonzalez and R. E. Woods, Digital Image Processing, Upper Saddle River, New Jersey, USA, 2008, ch. 5.

[5] R. H. Chan, C. W. Ho, and M. Nikolova, "Salt-and-pepper noise removal by median-type noise detectors and detail preserving regularization," IEEE Trans. Image Processing, vol. 14, no. 10, pp. 1479-1485, 2005.

[6] H. Ibrahim, K. C. Neo, S. H. Teoh, T. F. Ng, D. C. J. Chieh, and N. F. N. Hassan, "Impulse noise model and its variations," International Journal of Computer and Electrical Engineering (IJCEE), vol. 4, no. 5, pp. 647-650.

[7] M. E. Yuksel and M. T. Yildirim, "A simple neuro-fuzzy edge detector for digital images corrupted by impulse noise," AEU-International Journal of Electronics and Communications, vol. 58, no. 1, pp. 72-75, 2004.

[8] R. L. Swenson and K. R. Dimond, "A hardware FPGA implementation of a 2D median filter using a novel rank adjustment technique," in Seventh International Conference on Image Processing and Its Applications, vol. 1, 1999, pp. 103-106.

[9] H. Ibrahim, "Adaptive switching median filter utilizing quantized window size to remove impulse noise from digital images," Asian Trans. Fundamentals of Electronics, Communication \& Multimedia, vol. 2, no. 1, 2012.

[10] S. Zhang and M. A. Karim, "A new impulse detector for switching median filters," Signal Processing Letters, vol. 9, no. 11, pp. 360-363, 2002.

[11] S. H. Teoh and H. Ibrahim, "Median filtering frameworks for reducing impulse noise from grayscale digital images: A literature review," International Journal of Future Computer and Communication, vol. 1 , no. 4, pp. 323-326, 2012.

[12] R. Yang, L. Yin, M. Gabbouj, J. Astola, and Y. Neuvo, "Optimal weighted median filtering under structural constrains," IEEE Trans. Signal Processing, vol. 43, no. 3, pp. 591-604, 1995.

[13] Z. Wang and D. Zhang, "Progressive switching median filter for the removal of impulse noise from highly corrupted images," IEEE Trans. Circuits and Systems II: Analog and Digital Signal Processing, vol. 46, no. 1 , pp. 78-80, 1999.
[14] C. Spence and C. Fancourt, "An iterative method for vector median filtering," in Proc. IEEE International Conference on Image Processing (ICIP 2007), vol. 5, 2007, pp. 265-268.

[15] A. R. Forouzan and B. N. Araabi, "Iterative median filtering for restoration of images with impulse noise," in Proc. the $200310^{\text {th }}$ IEEE International Conference on Electronics, Circuits and Systems, vol. 1, pp. 232-235, 2003.

[16] G. R. Arce, "Statistical threshold decomposition for recursive and non-recursive median filters," IEEE Trans. Information Theory, vol. 32, no. 2, pp. 234-253, 1986.

[17] G. R. Arce and N.C. Gallagher Jr., "Stochastic analysis for the recursive median filter process," IEEE Trans. Information Theory, vol. 34, no. 4, pp. 669-679, 1988.

[18] A. Burian and P. Kuosmanen, "Tuning the smoothness of the recursive median filter," IEEE Trans. Signal Processing, vol. 50, no. 7, pp. 1631-1639, 2002.

[19] A. Nasimudeen, M. S. Nair, and R. Tatavarti, "Directional switching median filter using boundary discriminative noise detection by elimination," Signal, Image and Video Processing, vol. 6, no. 4, pp. 613-624, 2012.

[20] A. Hussain, M. A. Jaffar, and A. M. Mirza, "A hybrid image restoration approach: Fuzzy logic and directional weighted median based uniform impulse noise removal," Knowledge and Information Systems, vol. 24, no. 1, pp. 77-90, 2010.

[21] R. N. Czerwinski, D. L. Jones, and W. D. O. Brien Jr., "Ultrasound speckle reduction by directional median filtering," in Proc. International Conference on Image Processing, 1995, vol. 1, pp. 358-361.

[22] S. F. Liang, S. M. Lu, J. Y. Chang, and C. T. Lee, "A novel two-stage impulse noise removal technique based on neural networks and fuzzy decision," IEEE Trans. Fuzzy Systems, vol. 16, no. 4, pp. 863-873, 2008.

[23] V. Saradhadevi and V. Sundaram, "A novel two-stage impulse noise removal technique based on neural networks and fuzzy decision," International Journal of Computer Applications, vol. 21, no. 3, pp. 31-42, 2011.

[24] R. Kubota and N. Suetake, "Distribution distance-based threshold auto-tuning method for switching median filter," IEICE Electronics Express, vol. 7, no. 17, pp. 1310-1316, 2010.

[25] K. M. Moon, M. D. Patil, and B. Parmar, "Image restoration using adaptive switching median filter," in 2010 IEEE International Computational Intelligence and Computing Research (ICCIC), 2010, pp. 1-4.

[26] C. C. Hsieh, P. C. Huang, and S. Y. Hung, "Noisy image restoration based on boundary resetting BDND and median filtering with smallest window," WSEAS Transactions on Signal Processing, vol. 5, no. 5, pp. 178-187, 2009.

[27] P. E. Ng and K. K. Ma, "A switching median filter with boundary discriminative noise detection for extremely corrupted images," IEEE Trans. Image Processing, vol. 15, no. 6, pp. 1506-1516, 2006.

[28] K. K. Anisha and M. Wilscy, "Impulse noise removal from medical images using fuzzy genetic algorithm," Signal \& Image Processing: An International Journal (SIPIJ), vol. 2, no. 4, pp. 173-186, 2011.

[29] A. Toprak and I. Guler, "Suppression of impulse noise in medical images with the use of fuzzy adaptive median filter," Journal of Medical Systems, vol. 30, no. 6, pp. 465-471, 2006.

[30] A. Toprak, and I. Guler, "Impulse noise reduction in medical images with the use of switch mode fuzzy adaptive median filter," Digital Signal Processing, vol. 17, no. 4, pp. 711-723, 2007.

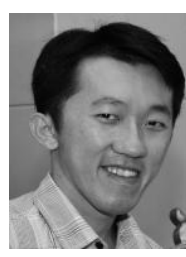

Sing Hoong Teoh received his B.Eng. degree in electronic engineering from Universiti Sains Malaysia, Malaysia, in year 2006. He is currently an engineer at Altera Malaysia, and furthering his MSc degree as a part time student at the School of Electrical and Electronic Engineering, Engineering Campus, Universiti Sains Malaysia, Nibong Tebal, Penang, Malaysia. His research interest is impulse noise reduction from digital images. 


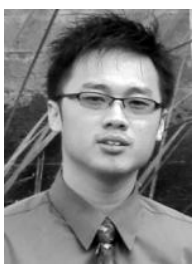

Boon Tatt Koik received his B.Eng. degree in electronic engineering from Universiti Sains Malaysia, Malaysia, in year 2012. He has published two research papers in international journals, regarding to digital image processing. His research interest includes digital image contrast enhancement, noise reduction, and change detection.

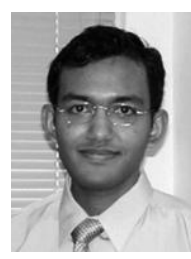

Haidi Ibrahim received his B. Eng degree in electronic engineering from Universiti Sains Malaysia, Malaysia, in year 2000. He then received his Ph.D. degree in digital image processing from the Centre for Vision, Speech, and Signal Processing (CVSSP), University of Surrey, Guildford, Surrey, United Kingdom in year 2005. He is now a senior lecturer at the School of Electrical and Electronic Engineering, Engineering Campus, Universiti Sains Malaysia, Nibong Tebal, Penang, Malaysia. His research interest includes digital image contrast enhancement, noise reduction, image segmentation, 3D visualization, and virtual reality. 